\title{
Heterogeneity of phenotype in two cystic fibrosis patients homozygous for the CFTR exon 11 mutation G551D
}

\author{
Richard B Parad
}

\begin{abstract}
In the heterozygous state, the cystic fibrosis transmembrane conductance regulator (CFTR) exon 11 mutation G551D has been described as "severe," causing pancreatic insufficiency. Two cystic fibrosis (CF) patients homozygous for this mutation showed a mild rather than severe pancreatic phenotype and a variable pulmonary phenotype.

$(\Im$ Med Genet 1996;33:711-713)
\end{abstract}

Key words: cystic fibrosis; CFTR exon 11; G551D.

CF has been associated with over 500 mutations in the CFTR gene. The most common mutation, $\Delta \mathrm{F} 508$, accounts for approximately $70 \%$ of $\mathrm{CF}$ alleles. G551D, an exon 11 mutation in the first nucleotide binding fold (NBF) of the CFTR protein, is one of the most common non- $\Delta \mathrm{F} 508$ mutations, occurring at a world wide frequency of $3 \%$. The influence of CFTR genotype on CF phenotype is poorly understood. Availability of only small numbers of patients homozygous for rare genotypes makes it difficult to accumulate enough clinical data to attempt phenotype correlation. Reported here are the clinical courses of two patients homozygous for CFTR mutation G551D. Potential pitfalls of using data available from heterozygotes to predict phenotype in homozygotes are illustrated.

\section{Division of \\ Respiratory Diseases, Ina Sue Perlmutter Cystic Fibrosis Research Laboratory, Children's Hospital, Harvard Medical School, 300 Longwood Avenue, Enders-1, Boston, MA 02115, USA. \\ R B Parad}

Received 27 December 1995 Revised version accepted for publication 15 March 1996

\section{Methods}

Cheekbrush DNA for CFTR mutation analysis was collected and prepared according to Richards et al. ${ }^{1}$ CFTR mutation analysis was performed for 12 mutations $(\Delta \mathrm{F} 508, \mathrm{G} 551 \mathrm{D}$, $\mathrm{G} 542 \mathrm{X}, 621+1 \mathrm{G} \rightarrow \mathrm{T}, \Delta \mathrm{I} 507,1717-1 \mathrm{G} \rightarrow \mathrm{A}$, R117H, N1303K, W1282X, R560T, R553X, $3849+10 \mathrm{~kb} C \rightarrow$ T). Genotyping for all CFTR mutations, except for $3849+10 \mathrm{~kb} \mathrm{C} \rightarrow \mathrm{T}$, which was assessed by restriction enzyme digestion with $H p h \mathrm{I},{ }^{2}$ were evaluated by the Multiplex Amplification Refractory Mutation System (ARMS). ${ }^{3}$ The reaction volumes were modified from the Ferrie protocol to incorporate $20 \mu \mathrm{l}$ of buccal cell DNA into the PCR reaction mixture. DNA from patient 1 was also assessed for 12 mutations by allele specific oligonucleotide analysis (ASO), ${ }^{4}$ but S549N was screened in addition, and $3849+10 \mathrm{~kb}$ was excluded.

\section{Case reports}

CASE 1

This patient was a white female diagnosed with CF at the age of 6 , with a history of recurrent pneumonia and no malabsorption or gastrointestinal symptoms. Family history was positive for CF in a maternal cousin. All grandparents were from Ireland, although there was no history of consanguinity. Quantitative pilocarpine iontophoresis at diagnosis showed a sweat chloride value of $107 \mathrm{mEq} / 1$. She was subsequently admitted to hospital two to three times a year for pulmonary exacerbations. She developed steroid dependent reactive airway disease. Her sputum grew mucoid Pseudomonas aeruginosa before the age of 12 . By the age of 15 , percent predicted forced vital capacity (FVC) and forced expiratory volume in one second $\left(\mathrm{FEV}_{1}\right)$ were $50 \%$. When pancreatic enzyme supplementation was started at the age of 16 (owing to low vitamin A level), height and weight were at the 50th centile for age. There was no liver disease or diabetes mellitus (DM).

In her mid teens she developed mild haemoptysis and gradually declining pulmonary function with increasing frequency of admission to hospital for pulmonary exacerbations. Chest radiograph and chest computed tomography showed bronchiectasis. By the age of 19 she required night time oxygen supplementation and developed $\mathrm{CO}_{2}$ retention. She died at the age of 20 of progressive respiratory failure.

CASE 2

This patient was first diagnosed with $\mathrm{CF}$ at the age of 36 with complaints of chronic bronchitis and asthma. Although always short and thin (height and weight at the 5th-10th centile), he had no malabsorption stools or GI symptoms until the age of 15 . At that time, he was admitted to hospital for a transient bowel obstruction which was successfully treated by enema. There was no liver disease or DM. Chronic cough was present from infancy. Although he complained of exertional dyspnoea as a child, asthma was not diagnosed until the age of 17 . Treatment included inhaled steroids and bronchodilators. There was no documented pneumonia and no admissions to hospital for respiratory problems. An infertility evaluation at the age of 33 showed azoospermia. His first episode of mild haemoptysis occurred at the age of 34 after a sudden coughing paroxysm. The CXR, which had been previously reported as unremarkable, had changed to a granular nodular pattern with peribronchial thickening. A diagnosis of sarcoidosis was considered, and 
thoracoscopic biopsy indicated an inflammatory non-granulomatous process and bronchitis. Because the diagnosis of CF was included in the differential diagnosis, a quantitative pilocarpine iontophoresis was performed and showed a sweat chloride of $101 \mathrm{mEq} / 1$. Sputum grew Staphylococcus aureus and rare nonmucoid Pseudomonas aeruginosa. Following a first course of intravenous antibiotics, postbronchodilator FVC was $98 \%, \mathrm{FEV}_{1} 70 \%$, and forced expiratory flow from 25 to $75 \%$ of vital capacity $\left(\mathrm{FEF}_{25-75}\right) 28 \%$. Vitamin A and E levels, prothrombin, and partial thromboplastin time and 72 hour stool fat collection were normal. Family history was negative for CF, and there was no known consanguinity.

\section{Results}

Fig 1 shows the multiplex ARMS reaction for assessment of mutations $\Delta \mathrm{F} 508, \mathrm{G} 542 \mathrm{X}$, $\mathrm{G} 551 \mathrm{D}$, and $621+1 \mathrm{G} \rightarrow \mathrm{T}$. The pair of lanes labelled 1 was performed on a known heterozygote for mutation G551D. The pair of lanes labelled 2 was performed on a non-CF control to illustrate a normal pattern. DNA from patient 1 showed a single band in the mutant lane for mutation G551D in the lanes under 3. This result was confirmed by ASO analysis (data not shown). The lanes under 4 show the same pattern seen in patient 2 . All other mutations tested for were absent.

\section{Discussion}

The CFTR missense mutation G551D is a Gly to Asp substitution at amino acid position 551 that results from a $G$ to $A$ substitution at the cDNA base pair numbered 1784 in exon $11 .^{5}$ This glycine is a highly conserved amino acid in the first nucleotide binding fold of the CFTR protein.

The allele frequency of G551D in CF patients from Europe has been documented in a north west to south east gradient, ${ }^{6}$ with the highest frequency reported in Northern Ireland $(8.3 \%)$ and less than $0.1 \%$ found in southern European/Mediterranean populations. In the USA, the frequency varies from 1 to $4 \%$. $^{\text {? }}$

Clinical utility would be derived from being able to anticipate CF disease severity given a patient's genotype. Rationalisations of how

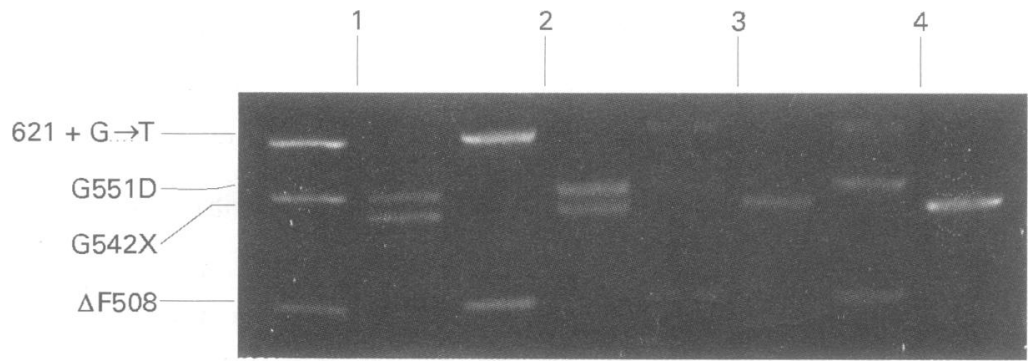

Figure 1 Ethidium bromide stained agarose gel showing PCR products of the multiplex $A R M S$ protocol. Lanes are paired to show both normal and abnormal alleles containing mutations $621+1 G \rightarrow T$ (normal band lane 1, abnormal band lane 2 ), G551D (abnormal band lane 1, normal band lane 2), G542X (abnormal band lane 1, normal band lane 2), and $\Delta F 508$ (normal band lane 1, abnormal band lane 2) at these loci. The first pair of lanes shows the ARMS pattern of a $G 551 D / N$ heterozygote. The band pattern of a normal subject (N/N) at these four loci is shown in the second pair of lanes. The third and fourth pairs of lanes were generated using DNA from patients 1 and 2 $(G 551 D / G 551 D)$ mutations could result in different severity of disease have been based on a number of schemes, including region of protein affected $(\mathrm{NBF} v$ transmembrane or regulatory domains), cellular function disrupted (protein production (category I) $v$ processing (category II) $v$ regulation (category III) $v$ conduction $\left(\right.$ category IV) ${ }^{8}$ ) or type of mutation (for example, missense $v$ frameshift or splice mutations). Genotype-phenotype correlations have been in closest agreement between pancreatic phenotype and specific mutations. Consistent pulmonary phenotype has only been suggested with two mutations, $\mathrm{R} 117 \mathrm{H}$ and $\mathrm{A} 455 \mathrm{E} .^{9}{ }^{10}$

G551D has been characterised as a class III mutation $^{8}$ through its presumed impact on ATP binding. ${ }^{11}$ Recent studies have shown present (but diminished) chloride conductance, and absent CFTR inhibitory regulation of the outwardly rectifying chloride channel. ${ }^{12}$ Organ specific phenotypes associated with individual CFTR mutations may in part be dependent on what back up systems are available for the disrupted CFTR function within the cells populating that organ.

G551D was originally categorised as a "severe" mutation with respect to pancreatic insufficiency (PI) ${ }^{13}$ All $21 \Delta$ F508/G551D heterozygotes, five compound heterozygotes for G551D and other non- $\Delta$ F508 mutations, and one G551D homozygote fit criteria for PI. "Severe" pancreatic alleles were also proposed to be recessive to "mild" pancreatic sufficient (PS) alleles, suggesting that a patient homozygous for a "severe" allele should present early symptoms of PI. In a retrospective cohort study by Hamash et al of $79 \Delta$ F508/G551D compound heterozygotes, no significant clinical differences from $\Delta \mathrm{F} 508$ homozygotes could be detected, with the exception of a lower risk for meconium ileus (MI) in the $\Delta \mathrm{F} 508 / \mathrm{G} 551 \mathrm{D}$ heterozygotes at birth. Of the other outcome parameters assessed, only the age at PI tended towards a later age in the compound heterozygotes $(1.9 \pm 2.8 v 2.7 \pm 4.0$ years $)$. Three G551D homozygotes were alluded to in that report, and they were briefly described with minimal detail as PI with no history of MI. A conflicting report by Curtis $e t a l^{14}$ documented three pancreatic sufficient G551D compound heterozygotes. Discrepancies in designation of compound heterozygotes as PS rather than mild PI could be based on differences in methods or criteria used for documenting that diagnosis.

For the two cases presented, phenotypic similarities include the relatively mild pancreatic (late mild PI in patient 1 compared to PS in patient 2) and mild intestinal phenotypes (no MI in the newborn period for either, and late meconium ileus equivalent in patient 2) and presentation with mainly respiratory symptoms (although one was mild and the other severe). The ages of diagnosis and age at $P I$ in patients 1 and 2 are much older than the means proposed for $\Delta \mathrm{F} 508 / \mathrm{G} 551 \mathrm{D}$ heterozygotes $^{6}$ (table 1$)$. The disparity in severity of respiratory disease of these patients could be related to the absence of mucoid Pseudomonas aeruginosa (an organism known to be associ- 
Table 1 Clinical characteristics: comparison between reported homozygotes for G551D and previously published heterozygous and homozygous series

\begin{tabular}{lccllll}
\hline Genotype & Ref & No of patients & Age at diagnosis $(y)^{*}$ & No with PIt & Age at PI $(y)^{*}$ & No with MIf \\
\hline Heterozygotes & & & & & & \\
G551D/4F508 & 5 & 79 & $1.9(2.8)$ & 79 & $2.7(4.0)$ & 5 \\
& 12 & 21 & NR & 21 & NR & 0 \\
G551D/other & 13 & 5 & NR & 5 & NR & 1 \\
Homozygotes & 12 & 3 & NR & 0 & NR & 0 \\
G551D/G551D & 5 & 1 & NR & 1 & NR & 0 \\
& 12 & 3 & NR & 3 & NR & 0 \\
& Patient 1 & 1 & 6 & 1 & 16 & 0 \\
& Patient 2 & 1 & 36 & 0 & NA & 0 \\
\hline
\end{tabular}

\footnotetext{
^ Mean (SD).

† Pancreatic insufficiency.

$\ddagger$ Meconium ileus.

NR, not reported

NA, not applicable.
}

ated with more rapid decline in pulmonary function) in sputum cultures from patient 2 .

The ARMS method of mutation analysis reported by Ferrie et al uses a built in control which requires, concurrent with the generation of a mutant amplimer by mutant primers, the absent production of normal amplimer by normal primers. In the homozygous mutant state, this is expressed as the appearance of a single band in the mutant lane. A band in both the mutant and normal lanes appears in the heterozygous state. Fig 1 shows that both patients 1 and 2 have only mutant bands and no normal bands for the amplimer containing the G551D mutation locus. Although the results in fig 1 are slightly faint for patient 1 (pair 3, lane 1), we have confirmed the result by a second mutation detection approach (ASO). Both methods suggest that there is only an abnormal, and no normal sequence at the G551D locus. It is still possible that a similar mutation, such as G551S, ${ }^{15}$ is present (rather than G551D) on one or both alleles and is producing a false positive result. Only sequencing at this locus would confirm the proposed genotype with certainty. Of course, the same criticism could be made of the genotypes reported for the other G551D carrying CF patients reviewed in table 1 . Another explanation for phenotype modification, assuming the common genotype of G551D/G551D, is the presence of a second mutation or sequence variant elsewhere in either or both alleles of the CFTR gene. ${ }^{16}$

The disparate outcomes of these two patients shows both that severe pancreatic insufficiency is not necessarily a clinical feature of the G551D homozygous genotype, and that a mild pulmonary phenotype is possible. Pulmonary outcome cannot be predicted by CFTR genotype alone. These findings support the hypothesis that considering the influence of non-CFTR genes and environmental influences will be necessary to understand variability in the CF phenotype.
This study was supported in part by $\mathrm{NIH}$ grant DK2273 and by a Trudeau Scholarship of the American Lung Association.

1 Richards B, Skoletsky J, Shuber AP, et al. Multiplex PCR amplification from the CFTR gene using DNA prepared from buccal brushes/swabs. Hum Mol Genet 1993;2:15963.

2 Highsmith WE, Burch LH, Zhou Z, et al. A novel mutation in the cystic fibrosis gene in patients with pulmonary disease but normal sweat chloride concentrations. $N$ Engl $\mathcal{F}$ Med 1994;331:974-80.

3 Ferrie RM, Schwarz MJ, Robertson NH, et al. Development, multiplexing, and application of ARMS tests for common mutations in the CFTR gene. Am f Hum Genet 1992;51:251-62

4 Shuber AP, Skoletsky J, Stern R, Handelin BL. Efficient 12-mutation testing in the CFTR gene: a general model for complex mutation analysis. Hum Mol Genet 1993; 2:153-8.

5 Cutting GR, Kasch LM, Rosenstein BJ, et al. A cluster of cystic fibrosis mutations in the first nucleotide-binding fold of the cystic fibrosis conductance regulator protein. Nature 1990;346:366-9.

6 Hamash A, King TM, Rosenstein BJ, et al. Cystic fibrosis patients bearing both the common missense mutation Gly-Asp at codon 551 and the delta F508 mutation are clinically indistinguishable from delta F508 homozygotes, except for decreased risk of meconium ileus. Am 7 Hum Genet 1992;51:245-50.

7 The Cystic Fibrosis Genetic Analysis Consortium. Population variation of common cystic fibrosis mutations. The Cystic Fibrosis Genetic Analysis Consortium. Hum Muta 1994;4:167-77.

8 Welsh MJ, Smith AE. Molecular mechanisms of CFTR chloride channel dysfunction in cystic fibrosis. Cell 1993;73:1251-4.

9 Cystic Fibrosis Genotype Phenotype Consortium. Correlation between genotype and phenotype in patients with cystic fibrosis. N Engl f Med 1993;329:1308-13.

$10 \mathrm{Gan} \mathrm{KH}$, Veeze HJ, Van Den Ouweland AMW, et al. A cystic fibrosis mutation associated with mild lung disease. $N$ Engl f Med 1995;333:95-9.

11 Logan J, Hiestand D, Daram P, et al. Cystic fibrosis transmembrane conductance regulator mutations that disrupt nucleotide binding. $\mathcal{F}$ Clin Invest $1994 ; 94: 228-36$.

12 Fulmer SB, Schwiebert EM, Morales MM, Guggino WB, Cutting GR. Two CFTR mutations have different effects on both pulmonary phenotype and regulation of outwardly rectified chloride currents. Pediatr Pulmonol 1995;suppl 12:182A

13 Kristidis P, Bozon D, Corey M, et al. Genetic determination of exocrine pancreatic function in cystic fibrosis. $A m \mathcal{F}$ Hum Genet 1992;50:1178-84.

14 Curtis A, Nelson R, Porteous M, Burn J, Bhattacharya SS Association of less common cystic fibrosis mutations with a mild phenotype. $\mathcal{F}$ Med Genet 1991;28:34-7.

15 Strong TV, Smit LS, Turpin SV, et al. Cystic fibrosis gene mutation in two sisters with mild disease and normal sweat electrolyte levels. N Engl f Med 1991;325:1630-4.

16 Kiesewetter S, Macek M Jr, Davis C, et al. A mutation in CFTR produces different phenotypes depending on chromosomal background. Nature Genet 1993;5:274-8. 